\title{
EFFECT OF ARONIA MELANOCARPA FRUIT JUICE ON CARRAGEENAN-INDUCED PAW EDEMA IN METABOLIC SYNDROME RATS
}

\author{
Mehmed Abtulov', Vasilena Kuzmanova ${ }^{2}$, Atanas Kuzmanov², Simeon Todorov², \\ Danail Pavlov ${ }^{3}$, Krasimir Kuzmanov ${ }^{4}$, Milena Todorova ${ }^{1}$, Miroslav Eftimov', \\ Silvia Gancheva ${ }^{1}$, Maria Zhelyazkova-Savova ${ }^{1}$, Stefka Valcheva-Kuzmanova ${ }^{1}$ \\ ${ }^{1}$ Department of Pharmacology and Clinical Pharmacology and Therapeutics, \\ Faculty of Medicine, Medical University of Varna \\ ${ }^{2}$ Student, Faculty of Dental Medicine, Medical University of Varna \\ ${ }^{3}$ Department of Biochemistry, Molecular Medicine and Nutrigenomics, \\ Faculty of Pharmacy, Medical University of Varna \\ ${ }^{4}$ Vivarium, Medical University of Varna
}

\section{ABSTRACT}

INTRODUCTION: Chronic, low-grade inflammation plays a critical role in the pathogenesis of the metabolic syndrome (MS). Aronia melanocarpa fruits are rich in biologically active compounds-polyphenols, which possess a variety of health benefits including an anti-inflammatory effect.

AIM: The aim of this article is to evaluate the effect of polyphenol-rich Aronia melanocarpa fruit juice (AMFJ) on carrageenan-induced acute inflammation in rats with diet-induced MS.

MATERIALS AND METHODS: Forty male Wistar rats were included in the experiment. They were allocated into 4 groups: $\mathrm{MS}, \mathrm{MS}+\mathrm{AMF} \mathrm{J}_{2.5}, \mathrm{MS}+\mathrm{AMFJ}_{5}$, and $\mathrm{MS}+\mathrm{AMFJ}_{10}$, all receiving high-fat high-fructose diet and $10 \%$ fructose in the drinking water for 10 weeks. The MS group served as a control and was treated daily with distilled water orally, while the other groups received AMFJ at doses of $2.5 \mathrm{~mL} / \mathrm{kg}, 5 \mathrm{~mL} / \mathrm{kg}$, and 10 $\mathrm{mL} / \mathrm{kg}$, respectively. At the end of the experiment, carrageenan was injected in the left hind paw in order to induce acute inflammation. Paw edema was evaluated with plethysmometer on the $30^{\text {th }}$ min and $1^{\text {st }}, 2^{\text {nd }}, 3^{\text {rd }}$, $4^{\text {th }}$, and $5^{\text {th }}$ hour after the injection.

RESULTS: In the MS group, the carrageenan-induced paw edema increased gradually with time reaching the highest value on the $5^{\text {th }}$ hour. A decrease throughout the whole 5 -hour period was observed in groups treated with AMFJ, the effect being most pronounced and statistically significant in MS+AMFJ ${ }_{5}$ group on the $2^{\text {nd }}$ and $3^{\text {rd }}$ hour.

CONCLUSION: Aronia melanocarpa fruit juice treatment in rats with MS resulted in a decrease in the carrageenan-induced paw inflammation. The anti-inflammatory effect might be attributed to the polyphenols in AMFJ.

Address for correspondence:

Mehmed Abtulov

Faculty of Medicine

Medical University of Varna

55 Marin Drinov St

9002 Varna

e-mail: Mehmed.Abtulov@mu-varna.bg

Received: September 1, 2021

Accepted: September 6, 2021
Keywords: Aronia melanocarpa, metabolic syndrome, rats, carrageenan, edema 


\section{INTRODUCTION}

Metabolic syndrome (MS) is a clinical condition affecting a significant percentage of the global population. It is a constellation of biochemical and clinical abnormalities (impaired levels of fasting glucose, triglycerides and HDL, central obesity, insulin resistance, elevated blood pressure), which increase the risk of developing atherosclerotic cardiovascular disease, and type 2 diabetes (1). Studies have shown that chronic, low-grade inflammation plays a critical role in the pathogenesis of the metabolic syndrome (2).

Carrageenan is a high molecular weight seaweed-derived sulfated polysaccharide commonly used as emulsifying agent in infant formulas, dairy products, and milk alternatives (almond milk) (3). It has been documented that carrageenan triggers an acute inflammatory response when injected subcutaneously. Carrageenan-induced rat paw edema is an experimental model used in preclinical studies in order to explore the anti-inflammatory potential of different molecules.

Aronia melanocarpa (black chokeberry) is an ornamental plant from the Rosaceae family, which was introduced in Europe in the early twentieth century. Due to the astringent taste, fresh fruits are usually used for the production of juice, nectar, wine, jam, dessert, tea, and herbal supplements (4). Aronia melanocarpa fruits are rich in polyphenolic compounds. Highest are the amounts of proanthocyanidins and anthocyanins (5). Polyphenols have been shown to possess a variety of health benefits due to their antioxidant, antiproliferative, and anti-inflammatory effects (6).

\section{AIM}

The purpose of the present study was to evaluate the effect of polyphenol-rich Aronia melanocarpa fruit juice (AMFJ) on carrageenan-induced acute inflammation in metabolic syndrome rats.

\section{MATERIALS AND METHODS}

\section{$A M F J$ Preparation and Determination of the} Content of Biologically Active Substances

The juice was prepared by grinding, pressing, and squeezing the fresh fruits grown in the Balkan Mountains, Bulgaria. It was filtered and preserved with potassium sorbate $(1.0 \mathrm{~g} / \mathrm{L})(7)$. The amount of total phenols, determined spectrophotometrically according to the procedure described by Folin-Ciocalteu (8) and presented as gallic acid equivalents (GAE), was $5461 \mathrm{GAE} / \mathrm{L}$. The amount of proanthocyanidins was $3122.5 \mathrm{mg} / \mathrm{L}$ and it was evaluated using the gravimetric method described by Howell et al. (9). The content of cyanidin glycosides and phenolic acids was determined by high-performance liquid chromatography and their contents were: cyanidin 3-galactoside $143.7 \mathrm{mg} / \mathrm{L}$, cyanidin 3-arabinoside $61.7 \mathrm{mg} / \mathrm{L}$, cyanidin 3-glucoside: $4.4 \mathrm{mg} / \mathrm{L}$, cyanidin 3-xyloside: $11.6 \mathrm{mg} / \mathrm{L}$; chlorogenic acid 585 $\mathrm{mg} / \mathrm{L}$, and neochlorogenic acid: $830 \mathrm{mg} / \mathrm{L}$.

\section{Animals and Experimental Protocol}

Forty male Wistar rats (initial body weight 160$280 \mathrm{~g}$ ) were included in the experiment. The animals were kept under 12/12 light-dark cycle, at an average ambient temperature of $20-25^{\circ} \mathrm{C}$ and had access to food and drinking water ad libitum. The animals were allocated into 4 groups (10 rats in each group): $\mathrm{MS}, \mathrm{MS}+\mathrm{AMFJ}_{2.5}, \mathrm{MS}+\mathrm{AMFJ}_{5}$, and $\mathrm{MS}+\mathrm{AMFJ}_{10}$. To induce MS, in the course of 10 weeks they were fed high-fat high-fructose (HFHF) diet consisting of lard (17\%) and fructose (17\%) with regular rat chow and received $10 \%$ fructose in the drinking water (10). During the whole experimental period, the $\mathrm{MS}+\mathrm{AMFJ}_{2.5}, \mathrm{MS}+\mathrm{AMFJ}_{5}$, and MS+AMFJ ${ }_{10}$ groups were respectively treated daily with AMFJ orally at doses of 2.5, 5 and $10 \mathrm{~mL} / \mathrm{kg}$. The MS group served as a control and received distilled water $(10 \mathrm{~mL} / \mathrm{kg})$. The experimental protocol is shown in Table 1.

All procedures concerning animal treatment and experimentation were conducted in conformity with the national and international laws and policies (EU Directive 2010/63/EU for animal experiments)

Table 1. Experimental protocol

\begin{tabular}{|c|c|c|}
\hline Group & Diet & Treatment \\
\hline MS & HFHF & $10 \mathrm{~mL} / \mathrm{kg}$ distilled water \\
\hline $\mathrm{MS}+\mathrm{AMFJ}_{2.5}$ & HFHF & $\begin{array}{l}2.5 \mathrm{~mL} / \mathrm{kg} \text { AMFJ diluted } \\
\text { with distilled water to a } \\
\text { total volume of } 10 \mathrm{~mL} / \mathrm{kg}\end{array}$ \\
\hline $\mathrm{MS}+\mathrm{AMFJ}_{5}$ & HFHF & $\begin{array}{c}5 \mathrm{~mL} / \mathrm{kg} \text { AMFJ diluted with } \\
\text { distilled water to a total } \\
\text { volume of } 10 \mathrm{~mL} / \mathrm{kg}\end{array}$ \\
\hline $\mathrm{MS}+\mathrm{AMFJ}_{10}$ & HFHF & $10 \mathrm{~mL} / \mathrm{kg}$ AMFJ \\
\hline
\end{tabular}


Mehmed Abtulov, Vasilena Kuzmanova, Atanas Kuzmanov et al.

Table 2. Values of the paw edema $(\mathrm{mL})$ on the subsequent time intervals; ${ }^{\star} p<0.05 v s$. MS group

\begin{tabular}{|c|c|c|c|c|c|c|}
\hline Group & $\begin{array}{c}\text { Paw Edema } \\
\left(30^{\text {th }} \text { minute }\right)\end{array}$ & $\begin{array}{c}\text { Paw Edema } \\
\left(1^{\text {st }} \text { hour }\right)\end{array}$ & $\begin{array}{l}\text { Paw Edema } \\
\left(2^{\text {nd }} \text { hour }\right)\end{array}$ & $\begin{array}{c}\text { Paw Edema } \\
\left.\text { ( } 3^{\text {rd }} \text { hour }\right)\end{array}$ & $\begin{array}{l}\text { Paw Edema } \\
\left.\text { ( } 4^{\text {th }} \text { hour }\right)\end{array}$ & $\begin{array}{c}\text { Paw Edema } \\
\left(5^{\text {th }} \text { hour }\right)\end{array}$ \\
\hline MS & $0.16 \pm 0.07$ & $0.37 \pm 0.08$ & $0.57 \pm 0.07$ & $0.71 \pm 0.07$ & $0.75 \pm 0.10$ & $0.87 \pm 0.09$ \\
\hline $\mathrm{MS}+\mathrm{AMFI}_{2.5}$ & $0.12 \pm 0.05$ & $0.17 \pm 0.06$ & $0.53 \pm 0.04$ & $0.65 \pm 0.04$ & $0.78 \pm 0.06$ & $0.70 \pm 0.12$ \\
\hline $\mathrm{MS}+\mathrm{AMFJ}_{5}$ & $0.20 \pm 0.01$ & $0.24 \pm 0.03$ & $0.36 \pm 0.03^{*}$ & $0.40 \pm 0.06^{*}$ & $0.62 \pm 0.08$ & $0.70 \pm 0.09$ \\
\hline $\mathrm{MS}+\mathrm{AMFJ}_{10}$ & $0.14 \pm 0.06$ & $0.19 \pm 0.06$ & $0.41 \pm 0.06^{*}$ & $0.40 \pm 0.10$ & $0.56 \pm 0.09$ & $0.73 \pm 0.07$ \\
\hline
\end{tabular}

and were approved by the Bulgarian Food Safety Agency (Document 177/07.07.2017).

\section{Induction of Paw Edema}

At the end of the treatment period, carrageenan at a dose of $1 \mathrm{mg}$ as $0.1 \mathrm{~mL}$ of freshly prepared solution in $0.9 \%$ saline was injected into the plantar surface of the left hind paw of the rats to induce acute inflammatory response. The paw volumes $(\mathrm{mL})$ were evaluated initially (before injection), on the $30^{\text {th }} \mathrm{min}$ ute and on the $1^{\text {st }}, 2^{\text {nd }} 3^{\text {rd }}, 4^{\text {th }}$, and $5^{\text {th }}$ hour after the injection by a digital plethysmometer LE7500 Panlab, Barcelona. The difference between the paw volumes before and after the injection correlated with the intensity of the inflammation induced.

The paw edema $(\mathrm{mL})$ was calculated using the following formula:

Paw edema $=\mathrm{V}_{\mathrm{s}}-\mathrm{V}_{0}$,

where: $V_{s}$-the paw volume measured at the six time intervals after the carrageenan injection, $\mathrm{V}_{0}-$ the initial paw volume.

$$
2^{\text {nd }} \text { hour }
$$

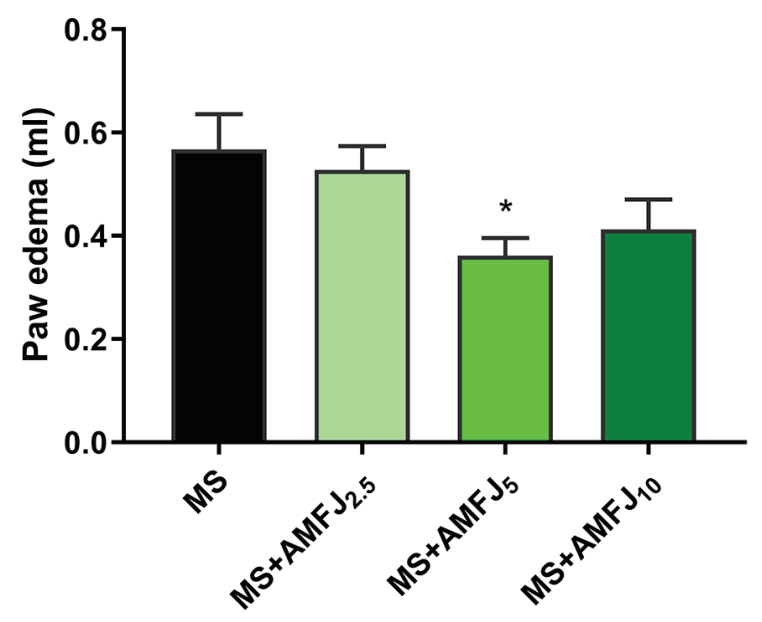

\section{Statistical Analysis}

GraphPad Prism 5.00 statistical software was used. The results were analyzed by one-way ANOVA and followed by Dunnett's multiple comparisons test. They were presented as mean \pm SEM and $\mathrm{p}<0.05$ was considered to indicate statistical significance.

\section{RESULTS}

Sub-plantar carrageenan injection induced an acute inflammation in all experimental groups. The values of the paw edema $(\mathrm{mL})$ after the injection are presented in Table 2. In the MS group, carrageenaninduced paw edema increased gradually with time reaching the value of $0.87 \pm 0.09 \mathrm{~mL}$ on the $5^{\text {th }}$ hour. A decrease of the paw edema throughout the whole 5-hour period was observed in groups treated with AMFJ, the effect being most pronounced and statistically significant in the MS+AMFJ ${ }_{5}$ group on the $2^{\text {nd }}$ and $3^{\text {rd }}$ hour and in the MS+AMFJ ${ }_{10}$ group on the $2^{\text {nd }}$ hour (Fig. 1).

Fig. 1. Rat paw edema $(m L)$ on the $2^{\text {nd }}$ (left) and $3^{\text {rd }}$ (right) hour after carrageenan injection; ${ }^{*} p<0.05 v s . M S$ 


\section{DISCUSSION}

Metabolic syndrome is considered as a lowgrade inflammatory state. This is associated with the visceral adipose tissue, which is a source of a number of cytokines, chemokines, hormonal elements, and various proteins. Some of these products have an anti-inflammatory activity (adiponectin), others-a pronounced pro-inflammatory (leptin, resistin, PAI1, CRP, IL-1, IL-6, TNF- $\alpha$, fibrinogen, serum amyloid A). In MS, the levels of pro-inflammatory mediators are elevated and those of anti-inflammatory mediators are decreased (11). Inflammatory molecules produced by visceral adipose tissue lead to a reduction in the insulin sensitivity, oxidative stress, endothelial dysfunction, and hypercoagulability. Taken together, all of these mechanisms contribute to the development of hypertension, hyperglycemia, and dyslipidemia, as well as other disorders associated with metabolic syndrome.

Carrageenan-induced paw edema is a commonly used model of acute inflammation. It consists of 2 phases: first phase (1-2 h after carrageenan injection) characterized by an increased release of serotonin, bradykinin, histamine from mast cells, and second phase (3-6 h after carrageenan injection) characterized by neutrophil infiltration, production of reactive oxygen species (ROS), as well as release of arachidonate metabolites such as prostaglandins, leukotrienes, and cytokine release (IL-1 $\beta$, IL-6, IL-10, and TNF- $\alpha)(12,13)$.

Aronia melanocarpa fruits are among the plant sources with the highest content of polyphenolic ingredients. Currently there are no data about the effects of Aronia melanocarpa fruit juice on carrageenan-induced edema in an experimental model of MS. This study examined the anti-inflammatory potential of polyphenol-rich AMFJ on carrageenan-induced paw inflammation in rats with MS. Aronia melanocarpa fruit juice caused a suppression in the acute inflammatory response during the six time intervals, having its highest values on the $2^{\text {nd }}$ (in $\mathrm{MS}+\mathrm{AMFJ}_{5}$ ) and $3^{\text {rd }}$ hour (in MS+AMFJ ${ }_{10}$ group) after carrageenan injection.

The effect on the $2^{\text {nd }}$ hour might be attributed to the ability of AMFJ to antagonize the effects of histamine, serotonin, and bradykinins. Such an effect of AMFJ has been well documented in a model of his- tamine-induced and serotonin-induced rat paw inflammation (14). In this study, the anti-inflammatory effect remained high during the $3^{\text {rd }}$ hour and was less pronounced thereafter. During the second phase of carrageenan-induced inflammation, AMFJ antagonized the effect of arachidonate metabolites and various cytokines. Such activities of Aronia polyphenols have been demonstrated in other studies. In a rat model of amiodarone-induced pulmonary toxicity, AMFJ administration decreased IL-6 (15). Dry Aronia melanocarpa extract (containing at least $25 \%$ anthocyanins) was found to inhibit markers of inflammation (IL-1 $\beta$, TNF- $\alpha$ ) and lipid peroxidation (malondialdehyde, MDA) in lipopolysaccharide-stimulated RAW 264 cells (16). Similarly, in a model of MS in rats induced by fructose-rich diet, Aronia extract supplementation resulted in a decrease of pro-inflammatory cytokines (IL-1 $\beta$, IL-6, TNF- $\alpha$ ) and increase of adiponectin (17). Athletes, supplemented with chokeberry juice, showed a decrease in the level of TNF- $\alpha$ and higher total antioxidant capacity (18). Black chokeberry supplementation in patients after myocardial infarction resulted in a decrease in the level of monocyte-chemoattractant protein-1 (MCP-1), C-reactive protein (CRP), IL-6, ICAM, and VCAM and an increase of the level of the anti-inflammatory adiponectin (19).

As described, carrageenan-induced paw inflammation is associated with oxidative stress. Based on that fact, we could assume that substances having an antioxidant effect would be beneficial. There are number of studies which demonstrate the antioxidant activity of Aronia melanocarpa polyphenols. Valcheva-Kuzmanova et al. found that AMFJ and its polyphenolic substances have catalase-like and superoxide dismutase-like effects and radical scavenging activity $(20,21)$. The juice used in this experiment was demonstrated to possess a high oxygen radical absorbance capacity (ORAC) and hydroxyl radical averting capacity (HORAC) in vitro (7). Polyphenolrich Aronia melanocarpa extracts significantly and dose-dependently inhibited the superoxide radical formation in patients at high cardiovascular risk (arterial hypertension, hypercholesterolemia, smoking, and diabetes mellitus) (22). In a model of 2,4,6-trinitrobenzenesulfonic acid (TNBS)-induced colitis, AMFJ improved the macroscopic and microscopic signs of colitis and prevented the increase in the con- 
Mehmed Abtulov, Vasilena Kuzmanova, Atanas Kuzmanov et al.

centrations of thiobarbirturic acid reactive substances (TBARS; marker for oxidative stress) in the colon (23). In a study by Coiocoiu et al. the antioxidant activity of polyphenol-rich Aronia melanocarpa extract was examined in an L-NAME-induced experimental model of arterial hypertension. A significant increase in the glutathione-peroxidase activity, total antioxidant capacity, as well as a significant decrease in the serum level of MDA was observed in Aroniatreated groups (24).

\section{CONCLUSION}

In rats with MS, AMFJ alleviated the carrageenan-induced paw inflammation. This effect might be attributed to the anti-inflammatory and antioxidant effects of the polyphenolic ingredients of the juice.

\section{REFERENCES}

1. Huang PL. A comprehensive definition for metabolic syndrome. Dis Model Mech. 2009;2(5-6):231-7.

2. Sharma P. Inflammation and the metabolic syndrome. Indian J Clin Biochem. 2011;26(4):317-8. doi: 10.1007/s12291-011-0175-6.

3. Martino JV, Van Limbergen J, Cahill LE. The role of carrageenan and carboxymethylcellulose in the development of intestinal inflammation. Front Pediatr. 2017;5:96. doi: 10.3389/fped.2017.00096.

4. Sidor A, Gramza-Michałowska A. Black chokeberry Aronia melanocarpa L.-A qualitative composition, phenolic profile and antioxidant potential. Molecules. 2019;24(20):3710. doi: 10.3390/ molecules 24203710.

5. Denev P, Č́ž M, Kratchanova M, Blazheva D. Black chokeberry (Aronia melanocarpa) polyphenols reveal different antioxidant, antimicrobial and neutrophil-modulating activities. Food Chem. 2019;284:108-17. doi: 10.1016/j. foodchem.2019.01.108.

6. Jurikova T, Mlcek J, Skrovankova S, Sumczynski D, Sochor J, Hlavacova I, et al. Fruits of Black chokeberry Aronia melanocarpa in the prevention of chronic diseases. Molecules. 2017;22(6):944. doi: 10.3390/molecules22060944.

7. Valcheva-Kuzmanova S, Denev P, Krachanova M, Surleva A, Belcheva A. Composition and antioxidant activity of Aronia melanocarpa fruit juice. Varna Med Forum. 2014;3(1):15-20. doi: 10.14748/ vmf.v3i1.1109.
8. Singleton VL, Rossi JA. Colorimetry of total phenolics with phosphomolybdic phosphotungstic acid reagents. Am J Enol Viticult. 1965;16(3):144-58.

9. Howell AB, Reed JD, Krueger CG, Winterbottom R, Cunningham DG, Leahy M. A-type cranberry proanthocyanidins and uropathogenic bacterial anti-adhesion activity. Phytochemistry. 2005;66(18):2281-91. doi: 10.1016/j. phytochem.2005.05.022.

10. Gancheva SM, Zhelyazkova-Savova MD, Galunska B, Chervenkov T. Experimental models of metabolic syndrome in rats. Scr Sci Med. 2015;47(2):14-21. doi: 10.14748/ssm.v47i2.1145.

11. Reddy P, Lent-Schochet D, Ramakrishnan N, McLaughlin M, Jialal I. Metabolic syndrome is an inflammatory disorder: a conspiracy between adipose tissue and phagocytes. Clin Chim Acta. 2019;496:35-44. doi: 10.1016/j.cca.2019.06.019.

12. Vinegar R, Truax JF, Selph JL, Johnston PR, Venable AL, McKenzie KK. Pathway to carrageenaninduced inflammation in the hind limb of the rat. Fed Proc. 1987;46(1):118-26.

13. Crunkhorn P, Meacock SC. Mediators of the inflammation induced in the rat paw by carrageenin. Br J Pharmacol. 1971;42(3):392-402. doi: 10.1111/ j.1476-5381.1971.tb07124.x.

14. Borissova P, Valcheva S, Belcheva A. Antiinflammatory effect of flavonoids in the natural juice from Aronia melanocarpa, rutin and rutin-magnesium complex on experimental model of inflammation induced by histamine and serotonin. Acta Physiol Pharmacol Bulg. 1994;20(1):25-30.

15. Valcheva-Kuzmanova S, Stavreva G, Dancheva V, Terziev L, Atanasova M, Dimitrova A, et al. Effect of Aronia melanocarpa fruit juice on amiodaroneinduced pneumotoxicity in rats. Pharmacogn Mag. 2014;10(38):132-40. doi: 10.4103/0973-1296.131024.

16. Banach M, Wiloch M, Zawada K, Cyplik W, Kujawski W. Evaluation of antioxidant and anti-inflammatory activity of anthocyanin-rich water-soluble Aronia dry extracts. Molecules. 2020;25(18):4055. doi: 10.3390/molecules25184055.

17. Qin B, Anderson RA. An extract of chokeberry attenuates weight gain and modulates insulin, adipogenic and inflammatory signalling pathways in epididymal adipose tissue of rats fed a fructoserich diet. Br J Nutr. 2012;108(4):581-7. doi: 10.1017/ S000711451100599X. 
18. Skarpańska-Stejnborn A, Basta P, Sadowska J, Pilaczyńska-Szcześniak L. Effect of supplementation with chokeberry juice on the inflammatory status and markers of iron metabolism in rowers. J Int Soc Sports Nutr. 2014;11(1):48. doi: 10.1186/ s12970-014-0048-5.

19. Naruszewicz M, Laniewska I, Millo B, Dłuzniewski M. Combination therapy of statin with flavonoids rich extract from chokeberry fruits enhanced reduction in cardiovascular risk markers in patients after myocardial infraction (MI). Atherosclerosis. 2007;194(2):e179-84. doi: 10.1016/j. atherosclerosis.2006.12.032.

20. Valcheva-Kuzmanova S, Gadjeva V, Ivanova D, Belcheva A. Antioxidant activity of Aronia melanocarpa fruit juice in vitro. Acta Alimentaria. 2007;34(4):425-8. doi:10.1556/AAlim.36.2007.4.5.

21. Valcheva-Kuzmanova S, Blagović B, Valić S. Electron spin resonance measurement of radical scavenging activity of Aronia melanocarpa fruit juice. Pharmacogn Mag. 2012;8(30):171-4. doi: 10.4103/0973-1296.96583.
22. Ryszawa N, Kawczyńska-Drózdz A, Pryjma J, Czesnikiewicz-Guzik M, Adamek-Guzik T, Naruszewicz $\mathrm{M}$, et al. Effects of novel plant antioxidants on platelet superoxide production and aggregation in atherosclerosis. J Physiol Pharmacol. 2006;57(4):611-26.

23. Valcheva-Kuzmanova S, Kuzmanov A, Kuzmanova V, Tzaneva M. Aronia melanocarpa fruit juice ameliorates the symptoms of inflammatory bowel disease in TNBS-induced colitis in rats. Food Chem Toxicol. 2018;113:33-9. doi: 10.1016/j.fct.2018.01.011.

24. Ciocoiu M, Badescu L, Miron A, Badescu M. The involvement of a polyphenol-rich extract of black chokeberry in oxidative stress on experimental arterial hypertension. Evid Based Complement Alternat Med. 2013;2013:912769. doi: $10.1155 / 2013 / 912769$. 